MATHEMATICS OF COMPUTATION

Volume 67, Number 222, April 1998, Pages 641-646

S 0025-5718(98)00948-X

\title{
NUMERICAL EVALUATION OF A SYMMETRIC POTENTIAL FUNCTION
}

\author{
LORI A. CARMACK
}

\begin{abstract}
We discuss the numerical evaluation of a symmetric potential function which arises naturally in applications. We present a method designed to accurately and efficiently compute this integral, and compare the performance of this method with two other popular techniques. This method requires considerably fewer function evaluations than all other techniques we tested, and is applicable to any integral which can be expressed in terms of complete elliptic integrals.
\end{abstract}

\section{INTRODUCTION}

The problem of computing potential functions occurs in a wide variety of applications including, but not limited to, fluid mechanics, acoustics, diffusion, and electromagnetics. Potential functions can prove troublesome numerically because of the possibility of singular integrands. Traditional methods such as Simpson's Rule or quadrature are therefore often computationally inefficient due to the large number of points required to achieve a reasonable amount of accuracy.

In this paper we consider the numerical evaluation of one particular potential function,

$$
\psi(r, z)=\frac{1}{2 \pi} \int_{0}^{\pi} \frac{\rho \cos \theta d \theta}{\left[r^{2}+\rho^{2}-2 r \rho \cos \theta+(z-\zeta)^{2}\right]^{\frac{1}{2}}},
$$

which arises naturally, for example, in the formulation of the axisymmetric incompressible Euler equations with swirl. We used a variety of different numerical evaluation techniques on this integral. We present here a method which computes this integral both accurately and efficiently, even when near a singularity in the integrand. Namely, we first express the integral in terms of complete elliptic integrals of the first and second kinds, and then use an algorithm found in Abramowitz and Stegan [1] to compute these elliptic integrals. We compare this technique to two other popular methods: Simpson's Rule, and a quadrature rule subroutine found in the book QUADPACK [5] designed specifically to handle integrals such as (1). Our method proved significantly superior to these other two. We proceed by first discussing the integral under consideration in further detail, and then present the numerical evaluation method. Last, with the aid of a table, we briefly present a comparison of the three techniques stated above.

Received by the editor August 21, 1996.

1991 Mathematics Subject Classification. Primary 31B99, 65D30, 76C99.

(C) 1998 American Mathematical Society 


\section{Derivation of ThE SYMMETRIC POTENTIAL FUNCTION}

The symmetric potential function (1) is derived by analytically solving Poisson's equation in three dimensions,

$$
-\Delta u=f
$$

using cylindrical coordinates, where $u(r, \theta, z)$ is of the form $e^{i \theta} \psi(r, z)$, and

$$
f(r, \theta, z)=e^{i \theta} \omega(r, z) .
$$

This problem arises in finding an analytic formula for the stream function of the axisymmetric incompressible Euler equations with swirl, for example, where $\psi(r, z)$ is the stream function, and $\omega(r, z)$ is the vorticity of the fluid. Solving for $u$ via convolution with the fundamental solution of the Laplacian along with a transformation to cylindrical coordinates yields

$$
u(r, \theta, z)=\frac{e^{i \theta}}{4 \pi} \int_{-\infty}^{\infty} \int_{0}^{\infty} \omega(\rho, \zeta)\left\{\int_{0}^{2 \pi} \frac{e^{i \lambda} d \lambda}{[h(r, z, \rho, \zeta)]^{\frac{1}{2}}}\right\} \rho d \rho d \zeta
$$

where we have defined

$$
h(r, z, \rho, \zeta)=r^{2}+\rho^{2}-2 r \rho \cos \lambda+(z-\zeta)^{2} .
$$

Substituting $e^{i \theta} \psi(r, z)$ for $u(r, \theta, z)$ and canceling $e^{i \theta}$ from both sides gives an analytic formula for $\psi$ :

$$
\psi(r, z)=\frac{1}{2 \pi} \int_{-\infty}^{\infty} \int_{0}^{\infty} \int_{0}^{\pi} \frac{\rho \omega(\rho, \zeta) \cos \lambda d \lambda}{\left[r^{2}+\rho^{2}-2 r \rho \cos \lambda+(z-\zeta)^{2}\right]^{\frac{1}{2}}} d \rho d \zeta .
$$

Finally, numerically integrating twice with respect to $\rho$ and $\zeta$ leaves us with the definite one-dimensional integral

$$
W(r, z, \rho, \zeta)=\frac{1}{2 \pi} \int_{0}^{\pi} \frac{\rho \cos \lambda d \lambda}{\left[r^{2}+\rho^{2}-2 r \rho \cos \lambda+(z-\zeta)^{2}\right]^{\frac{1}{2}}} .
$$

The above derivation is analogous to a common application in potential theory, where $N$ charged particles are given, located at points $x_{i}$ in two or three dimensions, with strengths $\kappa_{i}$. The goal is to compute the potential induced by these charges at some point $x$. This scenario leads to the potential function being the solution of the equation

$$
\Delta u=\sum_{i-1}^{N} \delta\left(x-x_{i}\right) \kappa_{i}
$$

For this reason, we will refer to a point $(r, z)$ as a "receiver," and to a point $(\rho, \zeta)$ as a "source."

\section{An EFFICIENT NUMERICAL EVALUATION TECHNiqUE}

There are two considerations when numerically evaluating the integral (3). First, we would like to achieve a sufficiently high order of accuracy. When the integrand contains oscillatory functions as in this case, the occurrence of round-off error is 
likely. Therefore special care must be taken when choosing a numerical evaluation technique. And second, we want the computation to be as efficient as possible by minimizing the number of function evaluations of the integrand. We present in this section a method for computing (3) which addresses both these issues.

The best approach we found for handling this integral is to first express it in terms of complete elliptic integrals of the first and second kind. Then using an algorithm found in Abramowitz and Stegun [1], we can compute these elliptic integrals efficiently to essentially any desired degree of accuracy.

The complete elliptic integrals are defined as:

$$
\begin{aligned}
K(m) & =\int_{0}^{\pi / 2} \frac{1}{\sqrt{1-m^{2} \sin ^{2} \theta}} d \theta, \\
E(m) & =\int_{0}^{\pi / 2} \sqrt{1-m^{2} \sin ^{2} \theta} d \theta,
\end{aligned}
$$

where $K(m)$ is the complete elliptic integral of the first kind, $E(m)$ the complete elliptic integral of the second kind, and $0 \leq m<1$.

We now express $W$ in terms of them. To do so, we first rewrite the denominator of the integrand:

$$
\begin{aligned}
r^{2}+\rho^{2}- & 2 r \rho \cos \lambda+(z-\zeta)^{2} \\
& =r^{2}+\rho^{2}+2 r \rho+(z-\zeta)^{2}-2 r \rho(1+\cos \lambda) \\
& =(r+\rho)^{2}+(z-\zeta)^{2}-2 r \rho\left(2\left(1-\sin ^{2} \frac{\lambda}{2}\right)\right) \\
& =(r+\rho)^{2}+(z-\zeta)^{2}-4 r \rho \cos ^{2} \frac{\lambda}{2}
\end{aligned}
$$

So we have that

$$
\begin{aligned}
W(r, z, \rho, \zeta) & =\frac{1}{2 \pi} \int_{0}^{\pi} \frac{\rho \cos \lambda d \lambda}{\left[r^{2}+\rho^{2}-2 r \rho \cos \lambda+(z-\zeta)^{2}\right]^{\frac{1}{2}}} \\
& =\frac{1}{2 \pi} \int_{0}^{\pi} \frac{\rho \cos \lambda d \lambda}{\left[(r+\rho)^{2}+(z-\zeta)^{2}-4 r \rho \cos ^{2} \frac{\lambda}{2}\right]^{\frac{1}{2}}} \\
& =\frac{1}{\pi} \int_{0}^{\pi / 2} \frac{\rho \cos 2 \theta d \theta}{\left[(r+\rho)^{2}+(z-\zeta)^{2}-4 r \rho \cos ^{2} \theta\right]^{\frac{1}{2}}} \\
& =\frac{1}{\pi} \frac{1}{\sqrt{(r+\rho)^{2}+(z-\zeta)^{2}}} \int_{0}^{\pi / 2} \frac{\rho \cos 2 \theta d \theta}{\sqrt{1-m^{2} \cos ^{2} \theta}}
\end{aligned}
$$

using the change of variable $\theta=\lambda / 2$ in the third equality above, and where

$$
m^{2}=\frac{4 r \rho}{(r+\rho)^{2}+(z-\zeta)^{2}} .
$$


Making the substitution $\theta=-\tau+\frac{\pi}{2}$ yields

$$
\begin{aligned}
& W(r, z, \rho, \zeta)=\frac{1}{\pi} \frac{\rho}{\sqrt{(r+\rho)^{2}+(z-\zeta)^{2}}} \int_{0}^{\pi / 2} \frac{\left(-1+2 \sin ^{2} \tau\right) d \tau}{\sqrt{1-m^{2} \sin ^{2} \tau}} \\
& =\frac{1}{\pi} \frac{\rho}{m^{2} \sqrt{(r+\rho)^{2}+(z-\zeta)^{2}}} \int_{0}^{\pi / 2} \frac{\left(-m^{2}+2 m^{2} \sin ^{2} \tau\right) d \tau}{\sqrt{1-m^{2} \sin ^{2} \tau}} \\
& =\frac{\rho}{\pi m^{2} \sqrt{(r+\rho)^{2}+(z-\zeta)^{2}}} \\
& \times \int_{0}^{\pi / 2} \frac{\left(2-m^{2}-\left(2-2 m^{2} \sin ^{2} \tau\right)\right) d \tau}{\sqrt{1-m^{2} \sin ^{2} \tau}} \\
& =\frac{2 \rho}{\pi m^{2} \sqrt{(r+\rho)^{2}+(z-\zeta)^{2}}} \\
& \times\left\{\int_{0}^{\pi / 2} \frac{\left(1-\frac{m^{2}}{2}\right) d \tau}{\sqrt{1-m^{2} \sin ^{2} \tau}}-\int_{0}^{\pi / 2} \sqrt{1-m^{2} \sin ^{2} \tau} d \tau\right\} .
\end{aligned}
$$

Then since

$$
\frac{2 \rho}{\pi m^{2} \sqrt{(r+\rho)^{2}+(z-\zeta)^{2}}}=\frac{\sqrt{\rho}}{\pi \sqrt{r} m}
$$

we finally have that

$$
W(r, z, \rho, \zeta)=\frac{\sqrt{\rho}}{\pi \sqrt{r} m}\left\{\left(1-\frac{m^{2}}{2}\right) K(m)-E(m)\right\} .
$$

To numerically compute the elliptic integrals $K(m)$ and $E(m)$, we use the standard process of the arithmetic-geometric mean (AGM) found for example in [1]. Starting with a given triple, $\left(a_{0}, b_{0}, c_{0}\right)$, successive triples $\left(a_{n}, b_{n}, c_{n}\right)$ are determined according to the iterative formulas:

$$
\begin{aligned}
a_{n} & =\frac{1}{2}\left(a_{n-1}+b_{n-1}\right), \\
b_{n} & =\left(a_{n-1} b_{n-1}\right)^{\frac{1}{2}}, \\
c_{n} & =\frac{1}{2}\left(a_{n-1}-b_{n-1}\right) .
\end{aligned}
$$

The procedure stops at the $n$th step when $a_{n}=b_{n}$, or in other words when $c_{n}<\varepsilon$ to the required tolerance.

To determine the values of the elliptic integrals $K(m)$ and $E(m)$ we start with the triple

$$
a_{0}=1, \quad b_{0}=\cos m, \quad c_{0}=\sin m
$$


and then compute the successive iterates $\left(a_{n}, b_{n}, c_{n}\right)$ to the desired degree of accuracy. We then use the formulas

$$
K(m)=\frac{\pi}{2 a_{n}}
$$

and

$$
\frac{K(m)-E(m)}{K(m)}=\frac{1}{2}\left(c_{0}^{2}+2 c_{1}^{2}+2^{2} c_{2}^{2}+\cdots+2^{n} c_{n}^{2}\right) .
$$

We remark that this procedure converges due to the fact that $m$ is strictly less than one since the inequality

$$
4 r \rho<(r+\rho)^{2}+(z-\zeta)^{2}
$$

implies

$$
m^{2}=\frac{4 r \rho}{(r+\rho)^{2}+(z-\zeta)^{2}}<1
$$

This technique for computing $W$ has the two advantages that the computation is relatively inexpensive since in practice $n$ is small (less than 10), and also we can be assured of achieving the required accuracy.

At this point it is important to mention that there are many other available techniques besides the AGM for computing elliptic integrals. The work of B. C. Carlson is especially noteworthy. He has performed extensive research in the design of algorithms for computing elliptic integrals, the most recent being [2]. Also many standard program libraries such as the commercially available NAG [4] and IMSL [3] libraries contain routines for elliptic integrals. It is the goal of future work to compare the numerical performance of these existing methods.

\section{Comparison With other methods}

We used a variety of different numerical integration/evaluation techniques on the integral (3). Here we compare the results of the AGM method with two others: Simpson's Rule and a quadrature rule. We will refer to these methods as SR, and QAWO, respectively. For the quadrature rule we used a numerical subroutine found in the book QUADPACK [5] which is designed specifically for integrands which are in the form of a function multiplied by an oscillatory function. We present the results of the comparison in the table below. We fixed a source point located at $(\rho, \zeta)=(.5,0)$, and varied receiver locations while achieving a fixed accuracy of $1 \times 10^{-12}$. In Column 2 we list the number $n$ required in the AGM algorithm to achieve this tolerance. In Columns 3 and 4 we record the number of function evaluations necessary to achieve the same tolerance for the SR and QAWO methods. The last column contains the computed value of the potential function $\psi$.

It is worth mentioning that as the receiver location nears the source location, the integrand becomes more singular. To compensate for this, the SR and QAWO methods require a significantly large number of function evaluations in order to maintain accuracy. It is especially in these instances that the AGM method proves considerably more efficient. 


\begin{tabular}{|r||r|r|r||c|}
\hline Receiver & AGM & SR & QAWO & $\psi(r, z)$ \\
\hline \hline$(.2,0)$ & 5 & 40 & 345 & .106680168537 \\
\hline$(.3,0)$ & 5 & 60 & 375 & .176487133092 \\
\hline$(.4,0)$ & 6 & 120 & 405 & .286062243649 \\
\hline$(.45,0)$ & 6 & 240 & 495 & .392176201305 \\
\hline$(.49,0)$ & 7 & 1120 & 1455 & .640156319987 \\
\hline$(.51,0)$ & 7 & 1140 & 1665 & .630628420410 \\
\hline$(.6,0)$ & 6 & 150 & 435 & .261741659375 \\
\hline$(.7,0)$ & 6 & 90 & 375 & .164670877661 \\
\hline$(.8,0)$ & 6 & 70 & 375 & .116903098115 \\
\hline$(.5, .3)$ & 5 & 60 & 345 & .117740821608 \\
\hline$(.5, .2)$ & 6 & 80 & 375 & .171073076875 \\
\hline$(.5,1)$ & 6 & 130 & 405 & .272789050148 \\
\hline$(.5, .05)$ & 6 & 250 & 435 & .380318726778 \\
\hline$(.5, .01)$ & 7 & 1130 & 765 & .635328849965 \\
\hline
\end{tabular}

\section{Concluding Remarks}

The technique of numerical integration/evaluation is in itself a difficult and intensive area of research. Specific techniques for numerically computing integrals often need to be designed on a case by case basis. We have presented here a numerical evaluation method which accurately and efficiently computes a symmetric potential function in hopes that this technique may prove useful, and that the ideas may be applied to other integrals which must be computed numerically.

\section{ACKNOWLEDGEMENT}

I express thanks to Thomas Sideris for help with this project.

\section{REFERENCES}

[1] M. Abramowitz and I. Stegun, Handbook of Mathematical Functions, Dover Publications, Inc., New York, 1965. MR 34:8606

[2] B. C. Carlson, "Numerical computation of real or complex elliptic integrals," Numer. Algorithms 10 (1995), no. 1-2, 13-26. MR 97h:33035

[3] IMSL Sfun/Library Users Manual (IMSL Inc., 2500 CityWest Boulevard, Houston, TX $77042)$.

[4] NAG Fortran Library (Numerical Algorithms Group, 256 Banbury Road, Oxford OX27DE, U. K. ), Chapter S.

[5] R. Piessens, et. al., QUADPACK, Springer-Verlag Berlin, New York, 1983. MR 85b:65022

Department of Mathematics, Ohio State University, Columbus, Ohio 43210

E-mail address: carmack@math.duke.edu

Current address: Department of Mathematics, Duke University, Durham, NC 27708 